\title{
LAS VARIABLES TEMPORALES EN EL ESPAÑOL DE COSTA RICA Y DE ESPAÑA: UN ESTUDIO COMPARATIVO
}

\author{
Sandra Schwab
}

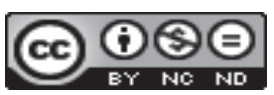

Esta obra está bajo una licencia Creative Commons

Reconocimiento-No Comercial-Sin Obra Derivada 



\title{
LAS VARIABLES TEMPORALES EN EL ESPAÑOL DE COSTA RICA Y DE ESPAÑA: UN ESTUDIO COMPARATIVO

\author{
THE TEMPORAL VARIABLES IN SPANISH SPOKEN IN COSTA \\ RICA AND IN SPAIN: A CROSS-LINGUISTIC STUDY
}

\author{
Sandra Schwab
}

\begin{abstract}
RESUMEN
El objetivo de esta investigación es describir las variables temporales en dos variantes del español: una de Costa Rica (San José) y la otra de España (Barcelona). De manera más específica, se examinan, con una perspectiva comparativa, la velocidad de elocución, el número de pausas, su duración y la velocidad de articulación en una tarea de lectura en voz alta. También se estudia el efecto de la edad y del sexo del locutor en la velocidad de articulación, así como el efecto de la longitud del enunciado, ya que se ha demostrado que estos factores afectan a la velocidad de articulación en otras lenguas como el francés, el inglés o el neerlandés. Los resultados principales del presente trabajo indican, por una parte, que los locutores costarricenses realizan pausas más largas que los locutores españoles y, por otra parte, que los locutores de las dos variantes presentan una velocidad de articulación similar, que se ve afectada por factores tanto extralingüísticos (i.e. la edad o la profesión de los locutores) como lingüísticos (i.e. longitud del enunciado).

Palabras clave: velocidad de elocución, velocidad de articulación, pausas, español de Costa Rica, español de España.
\end{abstract}

\begin{abstract}
This research aims at describing the temporal variables in two regional variants of Spanish: a variant from Costa Rica (San José) and a variant from Spain (Barcelona). More specifically, we examine, in a comparative perspective, the speech rate, the number and duration of pauses and the articulation rate in a reading task. The effect, on articulation rate, of the speakers' age and gender, as well as the effect of the utterance length are also investigated, given that such factors have been shown to affect articulation rate in other languages like French, English or Dutch. Results show that, on the one hand, Costa Rican speakers produce longer pauses than Spanish speakers, and on the other hand, speakers of the two variants present a similar articulation rate which is affected by extralinguistic factors (i.e. speakers' age or profession) as well as by linguistic factors (i.e. utterance length).
\end{abstract}

Key words: speech rate, articulation rate, pauses, Spanish, Costa Rica, Spain.

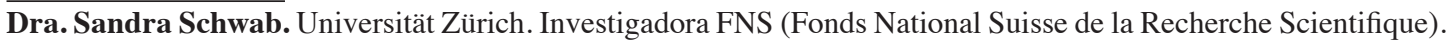
Université de Genève. Encargada de enseñanza. Didáctica de la fonética. Suiza.

Correo electrónico: sandra.schwab@unige.ch

Recepción: 12- 09- 2014

Aceptación: 13- 11- 2014 


\section{Introducción}

Grosjean y Deschamps (1972, 1973, 1975), al examinar la dimensión temporal del habla, proponen la noción de «variables temporales», entre las cuales se encuentran la velocidad de elocución, la velocidad de articulación y las pausas. La velocidad de elocución, que se basa tanto en el tiempo de articulación como en el tiempo de pausa, traduce la velocidad a la que un locutor produce un enunciado, mientras que la velocidad de articulación, que excluye el tiempo de pausa, refleja la velocidad a la que un locutor articula un enunciado. Tanto la velocidad de elocución como la velocidad de articulación pueden expresarse en sonidos por segundo, en sílabas por minuto (o en sílabas por segundo) o en palabras por minuto (véase Schwab en revisión, para una discusión acerca de las unidades usadas en el estudio de las variables temporales).

La velocidad de articulación -al igual que la velocidad de elocución, puesto que la segunda depende de la primera- se ve afectada por varios factores extralingüísticos y lingüísticos (Quené, 2008; Schwab, 2007). En cuanto a los factores extralingüísticos, se ha demostrado en diversas lenguas que la edad y el sexo del locutor tienen una influencia en la velocidad de articulación puesto que, por un lado, los locutores jóvenes articulan más rápidamente que los locutores mayores y, por otro lado, los hombres articulan más rápidamente que las mujeres (para el francés, Schwab y Avanzi, 2015; Schwab y Racine, 2012; para el inglés, Jacewicz et ál., 2009; para el neerlandés, Quené, 2008, Verhoeven et ál., 2004).

Respecto a los factores linguiísticos, la velocidad de articulación varía en función del estilo de habla. Siendo esto así, mientras que la lectura presenta, en unos estudios (por ejemplo, Grosjean y Deschamps, 1975), una velocidad de articulación más rápida que la conversación (i.e. habla espontánea), la tendencia inversa se observa en otros estudios (por ejemplo, Schwab y Avanzi, 2015; Crystal y House, 1990). Además, la longitud del enunciado tiene un impacto en la velocidad de articulación (Bartkova, 1991; Quené, 2008) ya que cuanto más largo es el enunciado, más rápida es la velocidad de articulación. Este efecto se conoce bajo el nombre de «anticipatory shortening» refiriéndose a que cuanto más sílabas anticipa el locutor en su enunciado, más tiende a acortar sus sílabas (Quené, 2008). La velocidad de articulación también puede depender de la variante lingüística (i.e. regional) que habla el locutor (por ejemplo, Schwab y Avanzi, 2015, para el francés; Jacewicz et ál., 2010, para el inglés; Quené, 2008 y Verhoeven et ál., 2004, para el neerlandés).

En español, tal y como mencionamos en Schwab (en revisión), las variables temporales (i.e. velocidad de elocución, velocidad de articulación y pausas) no han suscitado mucho interés. De hecho, hasta el momento son pocos los trabajos que las han investigado y los que las han abordado lo hacen en su mayoría indirectamente mientras examinan otro objeto de estudio.

Además, como todos estos trabajos no coinciden en la metodología seguida, resulta difícil definir las variables temporales del español y la influencia, en esta lengua, del conjunto de factores descritos previamente. No obstante, Schwab (en revisión), para facilitar una visión global, ofrece un inventario de los estudios que tratan sobre la velocidad de elocución en español, cuyo resumen se presenta en la Tabla 1. 
Tabla 1. Valores de velocidad de elocución y de articulación (expresadas en sílabas por segundo y en palabras por minuto), ordenados por velocidad de elocución (Tabla adaptada de Schwab, en revisión)

\begin{tabular}{|c|c|c|c|c|c|c|c|}
\hline \multirow[b]{2}{*}{ Autor } & \multirow{2}{*}{$\begin{array}{c}\text { Variante } \\
\text { lingüí́stica }\end{array}$} & \multirow{2}{*}{$\begin{array}{c}\text { Tarea } \\
\text { lingüística }\end{array}$} & \multirow[b]{2}{*}{$\mathbf{N}$} & \multicolumn{2}{|c|}{$\begin{array}{c}\text { Velocidad de } \\
\text { elocución }\end{array}$} & \multicolumn{2}{|c|}{$\begin{array}{l}\text { Velocidad de } \\
\text { articulación }\end{array}$} \\
\hline & & & & $\begin{array}{l}\text { Sílabas } \\
\text { por } \\
\text { segundo }\end{array}$ & $\begin{array}{c}\text { Palabras } \\
\text { por } \\
\text { minuto }\end{array}$ & $\begin{array}{c}\text { Sílabas } \\
\text { por } \\
\text { segundo }\end{array}$ & $\begin{array}{c}\text { Palabras } \\
\text { por } \\
\text { minuto }\end{array}$ \\
\hline $\begin{array}{l}\text { De Johnson et } \\
\text { ál. (1979) }\end{array}$ & México & Narración & 45 & 4.24 & 150 & 6.08 & 212 \\
\hline $\begin{array}{l}\text { García-Amaya } \\
(2009)\end{array}$ & Peninsular & Entrevista & 5 & 4.88 & 166.5 & - & - \\
\hline Blondet (2006) & Venezuela & Lectura & 4 & 5 & - & 6.1 & - \\
\hline $\begin{array}{l}\text { Madrid } \\
(2008)\end{array}$ & México & $\begin{array}{l}\text { Entrevista } \\
\text { especialista }\end{array}$ & 6 & 5.2 & 173.5 & 6 & 180.4 \\
\hline $\begin{array}{l}\text { Tapia-Ladino } \\
(1996)\end{array}$ & Peninsular & $\begin{array}{l}\text { Presentación } \\
\text { telediario }\end{array}$ & 4 & 6.65 & - & 7.28 & - \\
\hline $\begin{array}{l}\text { Tapia-Ladino } \\
\text { (1996) }\end{array}$ & Chile & $\begin{array}{l}\text { Presentación } \\
\text { telediario }\end{array}$ & 4 & 6.79 & - & 7.6 & - \\
\hline Rodero (2012) & Peninsular & $\begin{array}{l}\text { Presentación } \\
\text { telediario }\end{array}$ & 10 & 6.99 & 207 & - & - \\
\hline
\end{tabular}

Wainschenker et ál. (2002) se fijaron como meta definir una velocidad de elocución muy lenta, lenta, normal, rápida y muy rápida. Con este fin, usaron grabaciones de textos procedentes de diversas fuentes (por ejemplo, lecturas de artículos, conversaciones, noticiarios, reportajes, etc.) como estímulos en una prueba de percepción en la que los oyentes tenían que clasificar cada producción como muy lenta, lenta, normal, rápida y muy rápida.

Tabla 2. Valores de velocidad de elocución obtenidos en Wainschenker et ál. (2002)

\begin{tabular}{|c|c|c|c|c|c|c|c|c|}
\hline \multicolumn{2}{|c|}{ Velocidad de elocución } & Muy lenta & Lenta & Normal & Rápida & Muy rápida \\
\hline $\begin{array}{c}\text { Palabras por } \\
\text { minuto }\end{array}$ & Media & \multicolumn{2}{|c|}{39} & \multicolumn{2}{c|}{100} & \multicolumn{2}{c|}{152} & \multicolumn{2}{|c|}{208} & 287 \\
\cline { 2 - 9 } & Límites & \multicolumn{2}{|c|}{63} & 126 & 179 & 241 & \\
\hline
\end{tabular}

Como se puede ver en la Tabla 2, los autores establecieron valores límites inferiores y superiores para determinar cada velocidad y consideraron como normal una velocidad de elocución entre 126 y 179 palabras por minuto.

\section{Objetivo}

El objetivo de esta investigación es describir las variables temporales en dos variantes del español: la variante del español de Costa Rica (San José) y la variante de España (Barcelona). De manera más específica, examinamos, con una perspectiva comparativa, la velocidad de elocución, el número y la duración de las pausas y la velocidad de articulación, durante una tarea de lectura en voz alta. Igualmente, se estudia el efecto de la edad y del sexo del locutor, así como el efecto de la longitud del enunciado, ya que se ha demostrado que estos factores afectan a la velocidad de articulación en otras lenguas como son el francés, el inglés o el neerlandés. 


\section{Método}

\subsection{Participantes, material y procedimiento}

Dieciséis locutores hispanohablantes han participado en este experimento. Entre ellos, ocho (cuatro mujeres y cuatro hombres) provenían de la región de San José en Costa Rica (de ahora en adelante "CR") y ocho (cuatro mujeres y cuatro hombres) provenían de la región de Barcelona en España (a partir de ahora "ES"). La edad de los locutores no difería entre los costarricenses (media de 32 años, entre 20 y 52 años) y los españoles (media de 31.6 años, entre 19 y 55 años) $(\mathrm{F}(1,12)=0.003$, n.s. $)$, ni entre los hombres y las mujeres $(\mathrm{F}(1,12)=0.225$, n.s.), ni entre los hombres y las mujeres costarricenses y españolas $(\mathrm{F}(1,12)=0.061$, n.s.). Todos los locutores eran universitarios (estudiantes o empleados) de la Facultad de Letras de la Universidad de Costa Rica o de la Universitat Autònoma de Barcelona.

Los locutores costarricenses y españoles leyeron en voz alta, a una velocidad que consideraban como normal, una versión española del texto usado en el protocolo del proyecto "Phonologie du Français contemporain" (Durand et ál., 2009). La versión española, que se titula “¿El Primer Ministro irá a Béjar?”, contiene 21 frases y 366 palabras. Las grabaciones se efectuaron en salas dedicadas a este fin en las respectivas universidades, esto es, en la Universidad de Costa Rica en San José y en la Universitat Autònoma de Barcelona.

A continuación nos referimos a los "costarricenses" (CR) y a los "españoles" (ES), conscientes de que tanto los locutores de San José como los de Barcelona hablan una variante entre las muchas variantes que se hablan en su país de origen.

\subsection{Análisis de los datos}

Mediante el plugin EasyAlign (Goldman y Schwab, 2014) en Praat (Boersma y Weeninck, 2013), todas las producciones se alinearon automáticamente en sílabas y en palabras, comprobando manualmente las fronteras de las sílabas y de las palabras. Dada la fusión que se puede establecer entre vocales en español, fenómeno conocido como sinalefa (i.e. 2 vocales en hiato se pronuncian como una sola sílaba), no ha sido fácil decidir si una secuencia como "lleva al" se componía de tres sílabas (lle-va-al) o de dos (lle-val). Por esta razón, decidimos considerar las sílabas canónicas, tomando en cuenta, sin embargo, los eventuales errores o repeticiones para el recuento del número de sílabas (p. ej. "la la valoración" = 6 sílabas).

En cuanto a la identificación de las pausas, decidimos no definir un umbral de duración mínima para la duración de las pausas. Por lo tanto, todas las pausas, incluso las más cortas, fueron consideradas. Esta decisión fue motivada por la voluntad de no omitir las pausas cortas eventualmente realizadas a una velocidad rápida. Para diferenciar una verdadera pausa del silencio de una consonante oclusiva sorda, establecimos el procedimiento siguiente (aplicado a cada locutor): primero, medimos la duración del silencio de todas las oclusivas sordas en posición inicial de palabra (excepto cuando la palabra estaba precedida de una pausa de una duración de por lo menos $180 \mathrm{~ms}$ ). A partir de estas duraciones, a continuación, calculamos la media y la desviación típica, y determinamos un valor límite sumando 3 desviaciones típicas a la media. Por último, si la duración de un silencio de una 
oclusiva era superior al valor límite, este silencio se consideraba como una pausa y si era inferior, conservaba su estatus de silencio de oclusiva.

Una vez que las 16 producciones (8 locutores x 2 variantes) fueron alineadas en sílabas y en palabras y las pausas identificadas, realizamos tres análisis. En el primer análisis, examinamos la velocidad de elocución. Para ello, contamos el número total de sílabas y de palabras y medimos el tiempo total de locución. Calculamos, a partir de estos datos, la velocidad de elocución de cada locutor (en sílabas por minuto, en sílabas por segundo y en palabras por minuto). A continuación se proporcionan los datos en sílabas por segundo y en palabras por minuto para facilitar la comparación con estudios previos, pero sólo los datos en sílabas por minuto fueron analizados mediante un test-t no apareado en el que la variable dependiente era la velocidad de elocución y la variable independiente era la variante (CR vs. $\mathrm{ES} ; \mathrm{N}=8$ para cada variante).

En el segundo análisis, nos dedicamos al estudio de las pausas. Para ello, contamos el número de pausas producidas por cada locutor $(\mathrm{N}=8$ para cada variante) y medimos la duración (en ms) de cada una de las pausas. Las pausas superiores a $1500 \mathrm{~ms}(\mathrm{~N}=7)$ fueron descartadas. El número de pausas se analizó mediante un chi-cuadrado y con un test-t no apareado, mientras que la duración de las pausas se examinó mediante un modelo lineal mixto en el que el locutor se introdujo como variable aleatoria, la duración de las pausas como variable dependiente y la variante como variable independiente (Baayen et ál., 2008).

Finalmente, en el tercer análisis nos centramos en la velocidad de articulación. Por ello, contamos para cada grupo fónico (i.e. fragmentos de habla entre dos pausas) de cada locutor, el número de sílabas y de palabras y medimos el tiempo de articulación. Calculamos, a partir de estos datos, la velocidad de articulación (en sílabas por segundo y en palabras por minuto) de cada grupo fónico de los 16 locutores. Los grupos fónicos de menos de 3 sílabas fueron excluidos ( $\mathrm{N}=34 ; 3.72 \%$ de todos los grupos fónicos), lo que llevó a un total de 880 grupos fónicos (438 en los costarricenses y 442 en los españoles). Los datos en palabras por minuto se presentan para facilitar la comparación con los estudios anteriores, sin embargo sólo los datos en sílabas por segundo fueron analizados mediante un modelo lineal mixto en el que el locutor se consideró como variable aleatoria, la velocidad de articulación como variable dependiente y con las siguientes cuatro variables independientes: la variante, la edad, el sexo del locutor y el número de sílabas en el grupo fónico. Además de los efectos simples de las cuatro variables, nos interesaban las interacciones entre la variante y cada una de las otras variables, la interacción entre la edad y el sexo y la interacción triple entre la variante, la edad y el sexo.

\section{Resultados y discusión}

\subsection{Velocidad de elocución}

La Tabla 3 presenta los valores de velocidad de elocución para los locutores de Costa Rica y de España. Aunque aparezca que los costarricenses presentan una velocidad de elocución más lenta que los españoles, esta diferencia no alcanza el nivel de significación (en sílabas por minuto; $\mathrm{t}(14)=1.83, \mathrm{p}=0.09$ ). Por lo tanto, no se puede concluir que los costarricenses hablen más despacio que los españoles, aunque quepa tomar estos resultados con cautela, dado el número limitado de casos en cada variante. 
Según los valores presentados en las Tablas 1 y 2, tanto la velocidad de elocución de Costa Rica como la de España se encuentran en la categoría de una velocidad de elocución normal (i.e. entre 126 y 179 palabras por minuto según Wainschenker et ál., 2002). Blondet (2006), para la lectura de un texto producido por cuatro locutores venezolanos, propone una velocidad de elocución de 5 sílabas por segundo. Sin embargo, este valor se compara difícilmente por diferencias metodológicas con los valores que se observan en el presente trabajo (al igual que el valor de velocidad de articulación que se presentará más adelante).

Tabla 3. Valores medios de velocidad de elocución (y desviación típica) para los locutores de Costa Rica (CR) y de España (ES)

\begin{tabular}{|l|c|c|}
\hline Velocidad de elocución & $\begin{array}{c}\text { CR } \\
(\mathrm{N}=8)\end{array}$ & $\begin{array}{c}\text { ES } \\
(\mathrm{N}=8)\end{array}$ \\
\hline Sílabas por minuto & $319.02(26.66)$ & $349.85(36.17)$ \\
\hline Sílabas por segundo & $5.32(0.44)$ & $5.83(0.60)$ \\
\hline Palabras por minuto & $153.28(12.60)$ & $168.33(17.36)$ \\
\hline
\end{tabular}

\subsection{Número y duración de las pausas}

La Tabla 4 presenta el número y la duración de las pausas producidas por los locutores de Costa Rica y de España. Como se puede apreciar, tanto el número total como el número medio de pausas no difieren entre las dos variantes $\left(\chi^{2}(1)=0.01\right.$, n.s; $t(14)=0$, n.s., respectivamente).

Tabla 4. Número total, número medio (y desviación típica) y duración media (y desviación típica) de las pausas producidas por los locutores de Costa Rica (CR) y de España (ES)

\begin{tabular}{|l|c|c|}
\hline Número de pausas & $\begin{array}{c}\text { CR } \\
(\mathrm{N}=8)\end{array}$ & $\begin{array}{c}\text { ES } \\
(\mathrm{N}=8)\end{array}$ \\
\hline Total & 442 & 445 \\
\hline Media (desviación típica) & $55.25(10.00)$ & $55.63(11.44)$ \\
\hline Duración de las pausas (ms) & $\begin{array}{c}\text { CR } \\
(\mathrm{N}=442)\end{array}$ & $\begin{array}{c}\text { ES } \\
(\mathrm{N}=445)\end{array}$ \\
\hline Media (desviación típica) & $482(299)$ & $391(283)$ \\
\hline
\end{tabular}

En lo que concierne a la duración de las pausas, la Figura 1 presenta su distribución en los locutores de Costa Rica y de España. En las dos variantes, notamos, por un lado, la presencia de pausas inferiores a $150 \mathrm{~ms}$ (que se explica por no definir un umbral de duración mímina) y, por otro lado, la presencia de pausas superiores a $1000 \mathrm{~ms}$ (que constituyen pausas de respiración). A pesar de estas similitudes, los costarricenses producen, como lo indica la Tabla 2, pausas, en promedio, más largas que los españoles $(\mathrm{F}(1,864)=6.96, \mathrm{p}<.01)$. Por lo tanto, aparece que los costarricenses no producen pausas más numerosas que los españoles, sino pausas de duración más larga. 


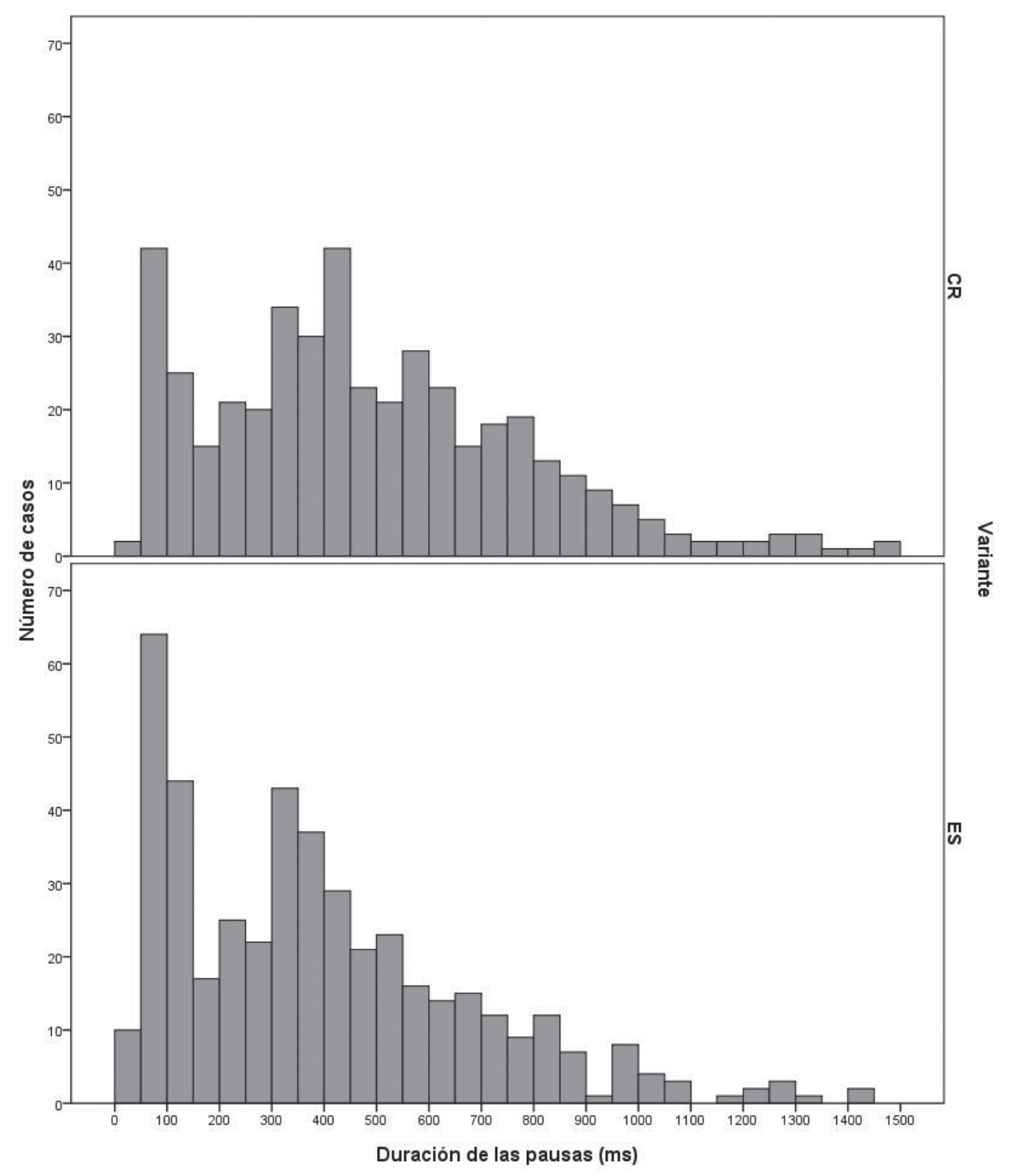

Figura 1. Duración de las pausas (en ms) producidas por los locutores de Costa Rica (CR) y de España (ES)

\subsection{Velocidad de articulación}

La Figura 2 presenta la distribución de la velocidad de articulación (en sílabas por segundo) para los locutores de Costa Rica (CR) y de España (ES). Como se puede notar, las dos distribuciones son muy similares, aunque la distribución de la velocidad de articulación de los españoles esté situada ligeramente más a la derecha en comparación con la de los costarricenses. Esta observación se ve reflejada en los valores, señalados en la Tabla 5, levemente más altos para los locutores de España que para los locutores de Costa Rica. 


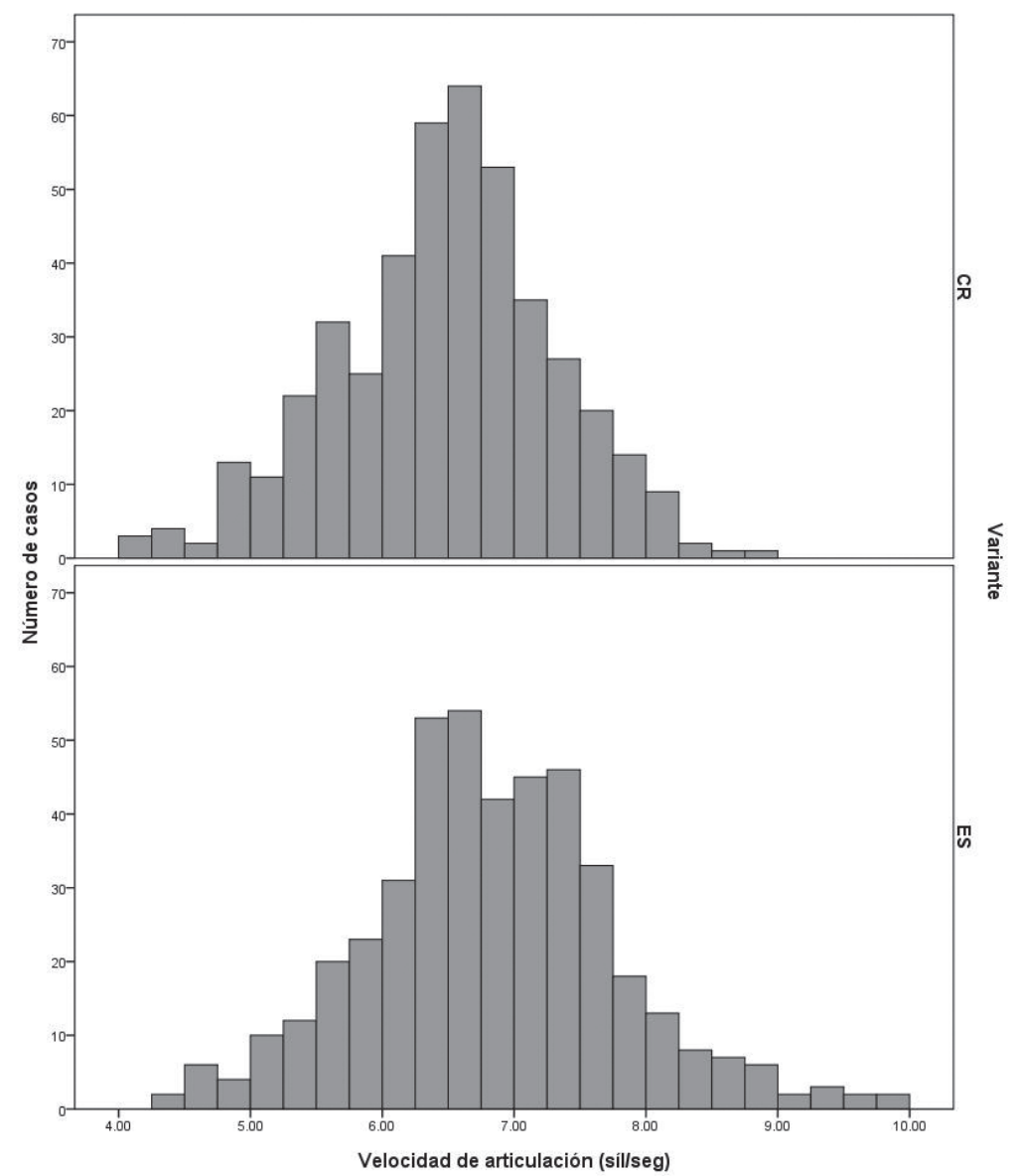

Figura 2. Velocidad de articulación (en sílabas por segundo) para los locutores de Costa Rica (CR) y de España (ES)

Tabla 5. Valores medios de velocidad de articulación (y desviación típica) para los locutores de Costa Rica (CR) y de España (ES)

\begin{tabular}{|l|c|c|}
\hline Velocidad de elocución & $\begin{array}{c}\text { CR } \\
(\mathrm{N}=438)\end{array}$ & $\begin{array}{c}\text { ES } \\
(\mathrm{N}=442)\end{array}$ \\
\hline Sílabas por segundo & $6.48(0.83)$ & $6.82(0.96)$ \\
\hline Palabras por minuto & $188.15(45.08)$ & $197.81(47.25)$ \\
\hline
\end{tabular}

No obstante, si examinamos los datos individuales de cada locutor (véase Figura 3), notamos que un locutor español particularmente rápido (ES8) podría ser responsable de la leve diferencia, que se acaba de mencionar, entre la velocidad de articulación en las dos variantes (véase Tabla 3), el resto de los locutores españoles presentando una velocidad de articulación parecida a la de los costarricenses. 


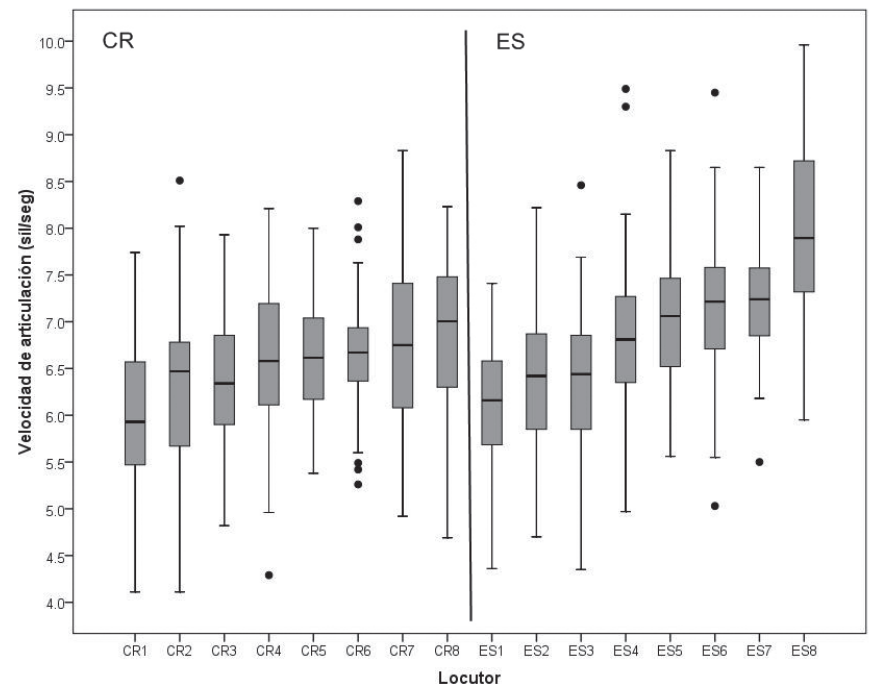

Figura 3. Velocidad de articulación (en sílabas por segundo) de los 8 locutores costarricenses (CR) y de los 8 locutores españoles (ES)

Para determinar si la ligera diferencia entre los costarricenses y los españoles era significativa, realizamos un análisis estadístico en el que, como ya se ha mencionado, no sólo examinamos el impacto de la variante sobre la velocidad de articulación, sino también el efecto del sexo y de la edad del locutor, así como el efecto del número de sílabas en el grupo fónico y las interacciones entre estas variables. Dado que, por una parte, la variable "Sexo" no tenía ningún efecto (ni interactuaba con las otras variables) y que, por otra parte, ninguna interacción era significativa, efectuamos un nuevo análisis sin la variable "Sexo" y sin interacciones.

Los resultados mostraron que la leve diferencia entre la velocidad de articulación de los costarricenses y de los españoles (en sílabas por segundo; véase Tabla 5) no alcanza significación estadística $(\mathrm{F}(1,858)=3.37, \mathrm{p}=.07)$. Por lo tanto, no se puede concluir que los costarricenses y los españoles articulen a una velocidad diferente.

De manera interesante, los resultados revelaron un efecto de la edad, independientemente de la variante $(\mathrm{F}(1,858)=6.91, \mathrm{p}<.01)$, pues, como se puede ver en la Figura 4 , la velocidad de articulación disminuye conforme aumenta la edad.

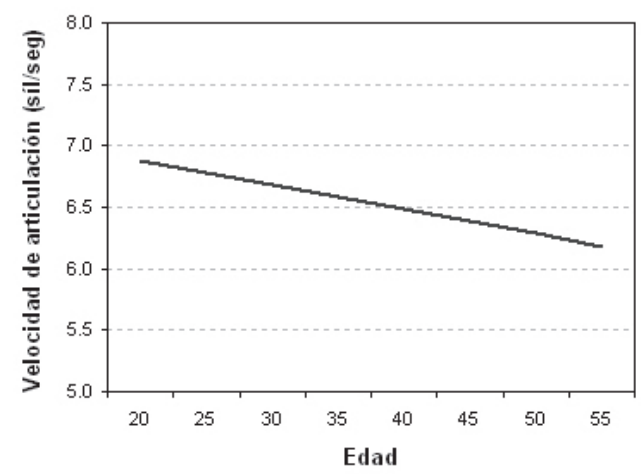

Figura 4. Velocidad de articulación (en sílabas por segundo) en función de la edad (CR y ES, agrupados) 
Finalmente, los resultados indicaron un efecto del número de sílabas en el grupo fónico, para cualquier variante $(\mathrm{F}(1,858)=130.84, \mathrm{p}<.001)$. Como se observa en la Figura 5, la velocidad de articulación aumenta conforme aumenta el número de sílabas en el grupo fónico.

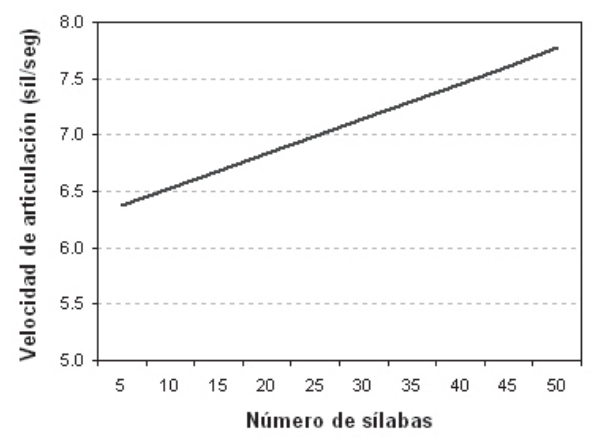

Figura 5. Velocidad de articulación (en sílabas por segundo) en función del número de sílabas en el grupo fónico (CR y ES, agrupados)

\section{Discusión general}

Esta investigación tenía como objetivo examinar la velocidad de elocución, el número y la duración de las pausas y la velocidad de articulación en español, más precisamente en dos variantes del español: una de Costa Rica (San José) y la otra de España (Barcelona).

En lo que respecta a la velocidad de elocución, la diferencia entre costarricenses y españoles no ha resultado significativa; sin embargo, el número limitado de casos en cada variante $(\mathrm{N}=8)$, al reducir el poder estadístico, nos obliga a considerar este resultado con precaución. En cuanto a las pausas, los resultados han mostrado que los costarricenses no producen más pausas que los españoles, sino pausas más largas.

Finalmente, respecto a la velocidad de articulación, los resultados indican que la diferencia entre costarricenses y españoles tampoco resulta significativa, esta vez con una gran cantidad de datos. Por lo tanto, se puede concluir que los costarricenses de San José y los españoles de Barcelona articulan con la misma velocidad.

Los estudios sobre la velocidad de articulación en francés, en inglés o en neerlandés (Schwab y Avanzi, 2015; Schwab y Racine, 2012; Jacewicz et ál., 2009; Quené, 2008; Verhoeven et ál., 2004) han mostrado una diferencia entre los hombres y las mujeres, puesto que los hombres articulan más rápidamente que las mujeres. No obstante, según nuestros datos, parece que esta diferencia no existe en español, cualquiera que sea la variante examinada. Sin embargo, la ausencia de diferencia entre la velocidad de articulación de los hombres y de las mujeres en español debe confirmarse con un número más importante de locutores.

También se ha demostrado en diversas lenguas que la velocidad de articulación disminuye conforme aumenta la edad. En aquellos estudios, los locutores se repartían o bien en dos grupos de edades (jóvenes y mayores) o bien a lo largo de un amplio continuum de edades. Por ejemplo, en francés, los locutores en Schwab y Avanzi (2015) tenían entre 19 y 86 años. En neerlandés, los participantes de Verhoeven et ál. (2004) se dividían entre locutores jóvenes (entre 21 y 40 años) y mayores (entre 45 y 59 años). En inglés, los participantes de Jacewicz et ál. (2009) eran 20 locutores jóvenes entre 20 y 34 años y 40 mayores entre 51 
y 65 años, mientras que los locutores de Jacewicz et ál. (2010) tenían entre 20 y 91 años. En nuestro estudio, sin embargo, el rango de las edades era menor puesto que los locutores tenían entre 19 y 55 años, con más locutores menores de 40 años $(\mathrm{N}=12)$ que mayores $(\mathrm{N}$ =4). No obstante, nuestros resultados mostraron un efecto de edad, con una velocidad de articulación más lenta para los locutores "mayores" (i.e. entre 40 y 55 años). En este caso, el efecto de la edad no puede explicarse por un déficit cognitivo o visual (como lo sugieren Jacewicz et ál., 2010), puesto que nuestros locutores "mayores" no eran tan mayores como para sufrir tales déficits, sino que se podría justificar por la profesión de los locutores. En efecto, los locutores "mayores" (tanto en Costa Rica como en España) eran profesores, por lo tanto, más acostumbrados, en comparación con los estudiantes, a leer, de manera inteligible, en voz alta. Esta conclusión subraya la necesidad de considerar, en el estudio de las variables temporales, variables sociales como la profesión para controlar, por ejemplo, el uso diario de la lectura en voz alta.

Finalmente, nuestro estudio indicó que el número de sílabas en el grupo fónico tiene un impacto sobre la velocidad de articulación (independiente de la variante): cuanto más largo es el grupo fónico, más rápida es la velocidad de articulación. Este resultado concuerda con las conclusiones a las que se ha llegado en francés (Schwab y Avanzi, 2015) y en neerlandés (Quené, 2008), pero no en inglés (Jacewicz et ál., 2010) y apoya la hipótesis de "anticipatory shortening”, es decir, al anticipar más sílabas en el grupo fónico, los locutores producen sílabas más cortas (i.e. una velocidad de articulación más rápida).

En conclusión, esta investigación revela, en primer lugar, que en lectura los locutores costarricenses realizan pausas más largas que los locutores españoles y, en segundo lugar, que los locutores de las dos variantes presentan una velocidad de articulación similar, que se ve afectada por factores tanto extralingüísticos (i.e. la edad o la profesión de los locutores) como lingüísticos (i.e. el número de sílabas en el grupo fónico). En el futuro, estas conclusiones deben ser validadas mediante estudios sobre el habla espontánea.

\section{Notas}

1. Le agradecemos a Ma Ángeles Barquero habernos proporcionado el texto PFC traducido al español, sus correcciones y sus sabios comentarios de una versión previa del manuscrito. También le damos las gracias a Claudia Peirano por el trabajo de verificación de la segmentación silábica. Esta investigación fue realizada parcialmente durante el período de subsidio Ambizione otorgado a Sandra Schwab por el Fondo Nacional Suizo para la Investigación Científica (PZ00P1_148036).

\section{Bibliografía}

Baayen, R. H. et ál. (2008). Mixed effects modeling with crossed random effects for subjects and items. Journal of Memory and Language. 59, 390-412.

Bartkova, K. (1991). Speaking Rate in French Application to Speech Synthesis. Proceedings of the 12th International Congress of Phonetic Sciences. Aix-en-Provence. (482-485).

Blondet, M. (2006). Variaciones de la velocidad de habla en español: patrones fonéticos y estrategias fonológicas. Un estudio desde la producción. (Tesis doctoral). Universidad de Los Andes.

Boersma, P. y Weenink, D. (2013). Praat, v. 5.3. http://www.fon.hum.uva.nl/praat/ [Consulta julio de 2014]. 
Collentine, J. et ál. (Eds.). (2009). Selected Proceedings of the 11th Hispanic Linguistics Symposium. Somerville, MA: Cascadilla Proceedings Project.

Congosto-Martín, Y. et ál. (Eds.). (en prensa). Fonética Experimental, Espacio Europeo Educación Superior e Investigación. Universidad de Cáceres y Universidad de Sevilla.

Crystal, T. H. y House, A.S. (1990). Articulation rate and the duration of syllables and stress groups in connected speech. Journal of the Acoustical Society of America. 88, 101112.

De Johnson, T. H. et ál. (1979). Temporal analysis of English and Spanish narratives. Bulletin of the Psychonomic Society. 13, 347-350.

Durand, J. et ál. (2009). Phonologie, variation et accents du français. Paris: Hermés.

García-Amaya, L. (2009). New findings on fluency measures across three different learning contexts. Por J. Collentine et ál. (Eds.). Selected Proceedings of the 11th Hispanic Linguistics Symposium. (68-80). Somerville, MA: Cascadilla Proceedings Project.

Gil, J. y Llisterri, J (Eds.). (en revisión). Fonética y fonología descriptivas de la lengua española. Madrid: Consejo Superior de Investigaciones Científicas.

Goldman, J. P. y Schwab, S. (2014). EasyAlign Spanish: an (semi-)automatic segmentation tool under Praat. Por Y. Congosto-Martín, M.L. Montero-Curiel y A. Salvador-Plans (Eds.). Fonética experimental, educación superior e investigación. (Vol. I, 629-640). Madrid: Arco/Libros.

Grosjean, F. y Deschamps, A. (1972). Analyse des variables temporelles du français spontané. Phonetica. 26, 129-157.

Grosjean, F. y Deschamps, A. (1973). Analyse des variables temporelles du français spontané II. Comparaison du français oral dans la description avec l'anglais (description) et avec le français (interview radiophonique). Phonetica. 28, 191-226.

Grosjean, F. y Deschamps, A. (1975). Analyse contrastive des variables temporelles de l'anglais et du français: Vitesse de parole et variables composantes, phénomènes d'hésitation. Phonetica. 31, 144-184.

Herrera, E. y Martín-Butragueño, P. (Eds.). (2008). Fonología instrumental. Patrones fónicos y variación. México: El Colegio de México.

Jacewicz, E. et ál. (2009). Articulation rate across dialect, age and gender. Language Variation and Change. 21, 233-256.

Jacewicz, E. et ál. (2010). Between-speaker and within-speaker variation in speech tempo of American English. Journal of the Acoustical Society of America. 128, 839-850.

Madrid, E. (2008). Hacia el establecimiento de unidades para la medición de la velocidad de habla. El caso del español. Por E. Herrera y P. Martín-Butragueño (Eds.). Fonología instrumental. Patrones fónicos y variación. (257-273). México: El Colegio de México.

Quené, H. (2008). Multilevel modeling of between-speaker and within-speaker variation in spontaneous speech tempo. Journal of the Acoustical Society of America. 123, 11041113. 
Rodero, E. (2012). A comparative analysis of speech rate and perception in radio bulletins. Text y Talk. 32, 391-411.

Schwab, S. (2007). Les variables temporelles dans la production et la perception de la parole. (Thèse de doctorat). Université de Genève.

Schwab, S. (en revisión). La velocidad de elocución en español. Por J. Gil y J. Llisterri (Eds.). Fonética y fonología descriptivas de la lengua española. Madrid: Consejo Superior de Investigaciones Científicas.

Schwab, S. y Avanzi, M. (2015). Regional variation and articulation rate in French. Journal of Phonetics. 48, 96-105.

Schwab, S. y Racine, I. (2012). Le débit lent des Suisses romands: mythe ou réalité? Journal of French Language Studies. 22, 1-15.

Tapia-Ladino, M. (1996). Velocidad y ritmo en el español público de Chile y España. (Tesis de Magister en Lingüística). Universidad de Concepción.

Verhoeven, J. et ál. (2004). Speech rate in a pluricentric language: A comparison between Dutch in Belgium and the Netherlands. Language and Speech. 47, 297-308.

Wainschenker, R. et ál. (2002). Medición cuantitativa de la velocidad del habla. Procesamiento del Lenguaje Natural. 28, 99-104. 
